\title{
Schema mediation in categorization learning
}

ED M. EDMONDS and SUSAN C. EDMONDS, Augusta College, Augusta, Ga. 30904

Subjects categorized unfamiliar stimuli representative of previously learned schema-defined categories significantly. better than chance. Categorization performance was not dependent upon knowledge of results. These findings are related to traditional views of concept learning and concept mediation.

Schema learning has been found to occur in a reproduction task (Edmonds \& Mueller, 1967a), a free sorting task (Evans \& Arnoult, 1967), and an oddity task (Edmonds \& Mueller, 1967b). In all these studies, Ss abstracted and used deviations from two or more different most probable column height sequences (schemata) to assign histoform patterns to their respective schema families (populations of patterns) without any external source of information.

Since schema learning has been clearly demonstrated, it is of interest to determine (1) if previously learned schemata can mediate transfer to unfamiliar stimuli which are representative of these schema families, and (2) the effects of knowledge of results on this categorization process. The present study used a transfer design to investigate these problems. In the training phase Ss attempted to classify patterns into two categories or schemata. In the testing phase these Ss were presented new patterns representing the two schemata. Thus, categorization performance significantly different from chance in the testing phase would support the conclusion that schemata mediate transfer to unfamiliar stimuli representative of previously learned classes of stimuli.

\section{SUBJECTS}

The Ss were 26 undergraduates enrolled at Augusta College. Each $\mathrm{S}$ was randomly assigned to one of two equal groups which are referred to as Knowledge of Results (KR) and no-KR (NKR).

\section{PATTERNS}

Forty VARGUS 7 patterns (Evans, 1967), 20 from each of two different schemata, were selected at $67 \%$ redundancy. These schemata had been used previously in other research (e.g., Edmonds \& Mueller, 1967a).

\section{PROCEDURE}

The Ss were told that they would be shown a number of different patterns which could be classified as representing either Pattern Class A or Pattern Class B. The Ss were further instructed to classify each pattern as either $A$ or $B$ and to record their responses on the provided. answer sheet.

In the training phase 15 patterns from each of the two schemata were presented in a randomized sequence. The testing phase consisted of the presentation of five new patterns from each schemata. Each pattern was projected onto a screen for $15 \mathrm{sec}$. The Ss were run in groups of four or less. After each pattern exposure, $S$ s were allowed $15 \mathrm{sec}$ to record their responses. The procedure was identical for both groups except that Group KR was informed of the correct response during the intertrial interval.

The performance of Group KR in the testing phase was assessed by counting the number of times $\mathrm{Ss}$ correctly categorized (A for Schema 1 and B for Schema 2) each pattern. This procedure was not appropriate for the NKR group, however, since $\mathrm{Ss}$ were free to assign the responses $\mathrm{A}$ and $B$ to either schema family. Therefore, performance data for these Ss were obtained by scoring the last 10 responses in terms of consistency with the categorization rule which the $S$ was using on the first 30 trials.

\section{RESULTS AND DISCUSSION}

The performance difference between Groups KR and NKR in the testing phase was nonsignificant although Group NKR categorized more patterns correctly than did Group KR. However, both groups performed significantly better than chance $(t=2.84, \mathrm{df}=12, \mathrm{p}<.02$ for Group KR).

These results indicate that $S$ can categorize new stimuli representative of previously learned schema-defined categories in the absence of any external information. In fact, KR does not appear to appreciably facilitate schematic concept formation in the categorization task as well as in certain other perceptual tasks (see Edmonds \& Mueller, 1968). Thus these findings concerning a schema as a mediating process are relevant to how new instances of a concept are categorized. It appears that this categorization process does not proceed by direct association through reinforcement but involves the discrimination of nonarbitrary attributes which relate a family of objects in the environment to each other.

The above statements should not be interpreted as implying that the conditions under which schematic concept formation (SCF) and schematic mediation occur without $\mathrm{KR}$ are unrestricted. Low redundancy levels (Brown, Walker, \& Evans, 1968), highly difficult tasks, and limited exposure to the schemata (Pearre \& Jones, 1968) are conditions that certainly will not enhance SCF (and indeed they should not). These conditions, however, will permit concept formation with KR. Moreover, many concepts can be acquired that are not even associated with schemata. The point we wish to make is simply that under certain conditions organisms can learn concepts defined by objects in the environment without any supplementary information. The findings of the present study further support this notion and clearly indicate the role of schemata as mediating processes.

\section{REFERENCES}

BROWN, B. R., WALKER, D. W., \& EVANS, S. H. Schematic concept formation as a function of constraint redundancy and knowledge of results. Psychonomic Science, 1968, 11, 75-76.

EDMONDS, E. M., \& MUELLER, M. R. Prediction of mixed schema learning in a reproduction task. Psychonomic Science, 1967a, 8, 533-534.

EDMONDS, E. M., \& MUELLER, M. R. The role of schemata in perceptual learning. Psychonomic Science, $19676,8,239-240$.

EDMONDS, E. M., \& MUELLER, M. R. Effects of incidental training and reinforcement on mixed schema learning. Psychonomic Science, $1968,10,75-76$.

EVANS, S. H. VARGUS 7: Computed pattems from Markov processes. Behavioral Science, 1967, 12, 323-328.

EVANS, S. H., \& ARNOULT, M. D. Schematic concept formation: Demonstration in a free sorting task. Psychonomic Science, 1967, 9, 221-222.

PEARRE, J. G., \& JONES, E. C. Effects of knowledge of results on schematic concept formation. Psychonomic Science, 1968, 12, 367-368.

NOTE

1. This research was supported in part by OE Grant No. 6-8338 to the senior author. 